\title{
Vascular Anatomy of the Cauda Equina and Its Implication on the Vascular Lesions in the Caudal Spinal Structure
}

\author{
Katsunari NAMBA ${ }^{1}$ \\ ${ }^{1}$ Center for Endovascular Therapy, Division of Neuroendovascular Surgery, \\ Jichi Medical University, Shimotsuke, Tochigi
}

\begin{abstract}
The cauda equina is composed of the lumbosacral and the coccygeal nerve roots and the filum terminale. In the embryonic period, discrepancy in development between the termination of the spinal cord and the spinal column results in elongation of the nerve roots as well as the filum terminale in this region. Although the vascular anatomy of the caudal spinal structure shares many common features with the other metameric levels, this elongation forms the basis of the characteristic vascular anatomy in this region. With the evolution of the high quality imaging techniques, vascular lesions in the cauda equina are being diagnosed more frequently than ever before. Albeit the demand for accurate knowledge of the vascular anatomy in this region, descriptions are often fragmented and not easily accessible. In this review, the author attempted to organize the existing knowledge of the vascular anatomy in the cauda equina and its implication on the vascular lesions in this region. Also reviewed is the clinically relevant embryological development of the cauda equina.
\end{abstract}

Key words: cauda equina, filum terminale, radicular, anatomy, vascular lesion

\section{Introduction}

The cauda equina comprises the lumbosacral, the coccygeal nerve roots, and the filum terminale. The vascular anatomy of the cauda equina is unfamiliar to us largely because the vessels are small and hardly visualized by radiological means. The infrequency of vascular lesions in this region is another reason for this. Although the vascular anatomy of the cauda equina is analogous to any spinal metameric level, the characteristic vascular anatomy in this region requires special attention. The vascular anatomy in this area has been extensively studied more than 30 years before using an injection method, and the legacy of these studies still provide the body of knowledge in this field. ${ }^{1-3)}$

With advancements in spinal and vascular imaging techniques, vascular lesions in the cauda equina are detected more frequently than in the past. However, because vascular lesions in this region are infrequent, and the anatomy is unique, understanding the pathology is not always easy. Therefore, in this era of improved magnetic resonance imaging/ magnetic resonance angiography (MRI/MRA) quality and high resolution spinal angiography, accurate

Received January 6, 2016; Accepted January 28, 2016 knowledge of the vascular anatomy in the cauda equina is required more than ever before. This review aims to revisit and organize the past works on the vascularization of the cauda equina, and then discuss its implication on the vascular lesions in this region. The relevant embryological development of the caudal spinal cord will be reviewed as well.

\section{Development of the Caudal Spinal Cord}

The development of the caudal spinal cord is distinct from its rostral counterpart, and may have an unknown but significant influence on the pathology of the vascular lesions in the caudal spinal structure. This will be discussed briefly in this section. The development of the nervous system involves two processes called the primary and secondary neurulation. Neurulation is the word to describe the formation of the neural tube. The primary neurulation begins with the elevation of the lateral edges of the neural plate to form the neural folds. The fusion of the neural folds results in the formation of the neural tube, the future spinal cord, and the brain.4) Following the fusion of the caudal region of the neural tube (posterior neuropore), the secondary neural tube begins to develop by elongation and cavitation of the tail bud..5) The tail bud is an aggregate of undifferentiated mesodermal cells at the 
caudal end of embryos, which can potentially give rise to multiple organs including the tail gut, coelom, blood vessels, notochord, somites, and spinal cord. ${ }^{6}$ ) The secondary neural tube eventually connects with the primary neural tube to form the posterior neural tube. ${ }^{7)}$ This posterior neural tube develops into the future lumbar, sacral, coccygeal, and equinal cord. Failure of the primary and secondary neurulation has been implicated as the cause of neural tube defects. $\left.{ }^{8}\right)$ Likewise, although not reported to date, this unique style of the posterior neural tube development may affect the pathogenesis of the vascular lesions in the caudal spinal area.

The development of the filum terminale requires special attention in order to understand the vascular anatomy of the structure and its disease. In the early twentieth century, pivotal studies on the development of the filum terminale were conducted by Kunitomo ${ }^{9)}$ and Streeter. ${ }^{10)}$ In their studies, the caudal end of the spinal cord in young embryos possessed distinct ependymal, mantle, and marginal zones; a feature of a well-developed spinal cord. Whereas, in a later period, with further development, the mantle zone disappeared leaving only the ependymal tube covered with a thin layer of marginal or fibrous layer. The spinal cord appeared much primitive than in the earlier period, and thus, this form of development process was called "dedifferentiation" by the author. The coccygeal portion that had undergone dedifferentiation represented the future filum terminale. Therefore, from the embryological point of view, the filum terminale can be considered to be the extension of the spinal cord. This view is supported by the anatomical finding that the central canal of the spinal cord extends into the filum terminale to become the ependymal lined ventriculus terminalis. ${ }^{11)}$ Histologic studies demonstrating neural and glial tissues in the filum terminale further reinforce this view. ${ }^{11-13)}$

\section{Arterial Anatomy of the Cauda Equina}

In this section, the arterial supply to the cauda equina structures will be reviewed. From the embryological point of view, the filum terminale is a part of the spinal cord, whereas the nerve roots belong to the peripheral nervous system. Therefore, the vascular structure of the filum terminale and the cauda equina nerve roots differ significantly, and will be discussed separately.

\section{Arterial supply to the extradural cauda equina nerve roots}

The arterial supply to the cauda equina can be divided into the L1-L4 lumbar region and L5 to sacrococcygeal region. In the L1-L4 region, the corresponding lumbar arteries give rise to the radicular artery of the cauda equina as in any other metameric level. The arterial supply to the L1-4 region is similar to the cervical and thoracic spinal nerve root arterial anatomy, and will not be discussed in detail. In the L5 and sacrococcygeal region, the arterial supply also follows the metameric distribution. However, the rich anastomosis in this area confers wide diversity to the vascular pattern in this area. The arterial supply to the cauda equina nerve roots is dependent on the hemodynamic balance among the middle sacral, iliolumbar, and lateral sacral arteries (Fig. 1).

The middle sacral artery arises from the caudal end of the abdominal aorta at the dorsal surface (Figs. 1, 2; large arrows). The artery gives rise to a pair of the lowest lumbar artery, which is the

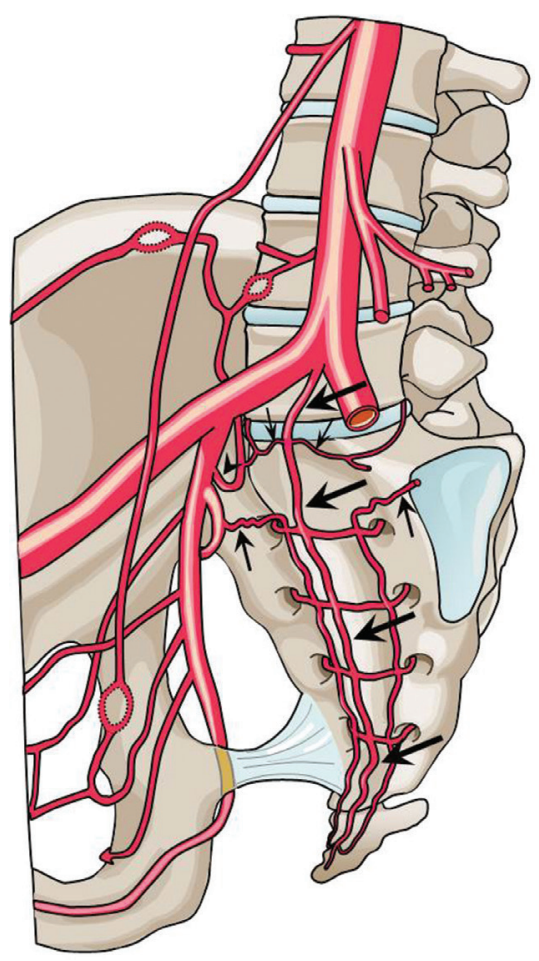

Fig. 1 A schema showing the arterial supply to the lumbosacral and coccygeal spinal structures. The middle sacral artery (large arrows) gives rise to a pair of the lowest lumbar artery (small arrows), which is the L5 segmental artery. A branch of the iliiolumbar artery (arrowhead), may also supply the L5 region. The size of this vessel is inversely related to the development of the lowest lumbar artery. The spinal structures within the sacral vertebral body are usually supplied by the branches of the lateral sacral arteries (arrows), which course medial to the anterior sacral foramen. The branches of the sacral artery form numerous anastomoses with the lateral branches of the medial sacral artery. 


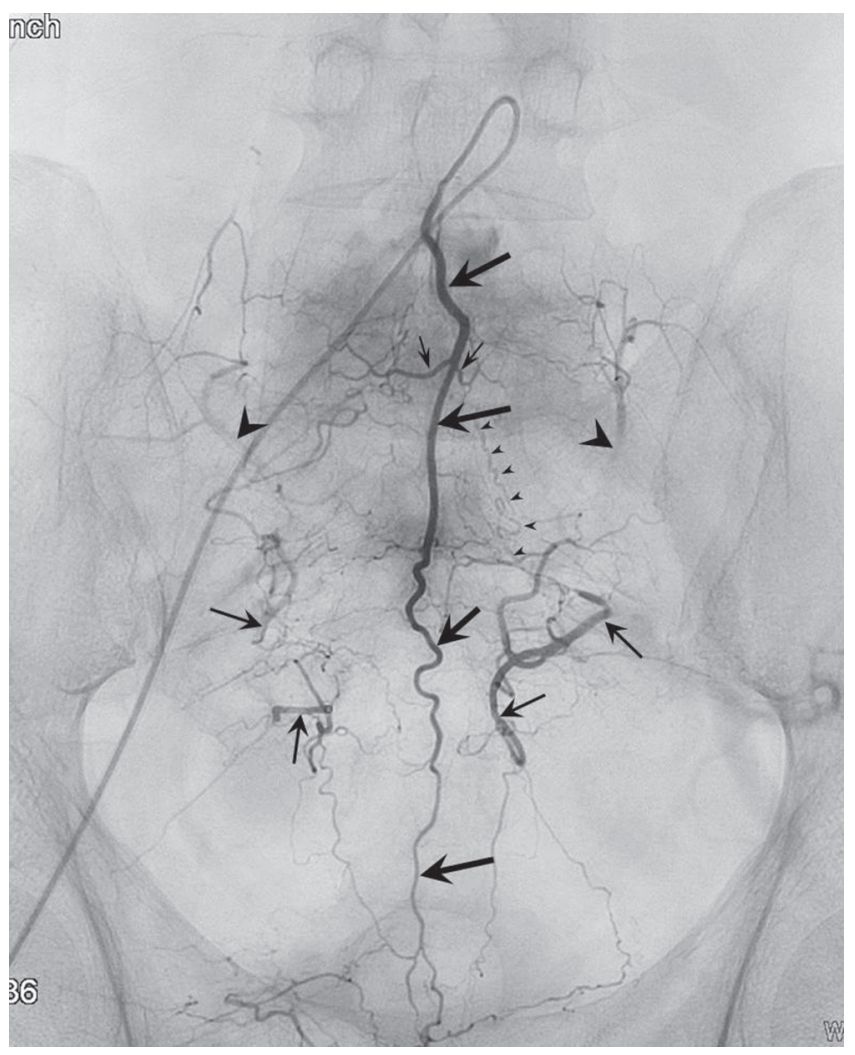

Fig. 2 An angiogram of the medial sacral artery (large arrows) demonstrates the arterial network of the lumbosacral and coccygeal region. A pair of the lowest lumbar artery is rising from the medial sacral artery (small arrows). Retrograde opacification of the iliolumbar arteries (arrowheads), lateral sacral arteries (arrows) are seen bilaterally. Note the opacification of a pathologic S1 radicular artery (small arrowheads) supplying an arteriovenous fistula in a higher level (not shown) that originates from the lateral sacral artery.

L5 segmental artery (Figs. 1, 2; small arrows). The middle sacral artery also gives rise to pairs of sacral branches that supply each sacral segment. The branches anastomose with the right and left lateral sacral arteries that lie on the anterior surface of the sacrum, medial to the sacral foramen (Figs. 1, 2; arrows).

The iliolumbar artery is usually the first branch of the internal iliac artery and arises from the dorsal surface (Figs. 1, 2; arrowheads). The arterial territory of the iliolumbar artery overlaps with that of the lowest lumbar artery, and is in reciprocal relationship with the development of the vessel. The iliolumbar artery gives rise to a lumbar branch that travels to the intervertebral foramen between the L5 and S1 vertebrae.

The lateral sacral artery arises from or shares a common trunk with the superior gluteal artery and travels caudally on the anterior surface of the sacrum, medial to the anterior sacral foramen (Figs. 1, 2; arrows). The lateral sacral artery gives rise to five pairs of medial and lateral braches. The medial branches form a network of vessels and anastomose with the middle sacral artery. The lateral branches reach the anterior sacral foramen and supply the structures within the sacral canal.

\section{Arterial supply to the intradural cauda equina nerve roots}

Anterior and posterior radicular arteries penetrate the supero-lateral aspect of the dural sheath of each lumbar and sacral spinal nerve just proximal to its dorsal root ganglion. They run uninterrupted courses along the respective anterior and posterior nerve filaments of the cauda equina to join arteries on the surface of the spinal cord. ${ }^{2)}$ Crock et al. published a comprehensive textbook concerning the normal vascularization of the cauda equina. In their publication, using a vascular injection method, they clearly visualized that all the anterior and posterior nerve root filaments are accompanied by a radicular artery. The anterior radicular arteries run cranially and eventually join either the anterior spinal artery (Fig. 3 right, short arrows) or the adjacent vasa corona (Fig. 3 right, arrowheads). Similarly, the posterior radicular arteries are cranially continuous with the posterior spinal artery (Fig. 3 left, short arrows) or the vasa corona (Fig. 3 left, arrowheads). Fig. 3, which was interpreted and reproduced from the study of Crock et al., illustrates that the arterial supply to the cauda equina and conus medullaris is segmental as well. Discussions concerning whether these arteries are functionally effective in supplying the spinal cord or Crock's observations are contradictory to the classical opinions that the spinal cord is supplied by a few principal radicular arteries are intriguing, but beyond the scope of this review. ${ }^{114-17)}$ However, one must know that there certainly exists a potential anastomotic channel between the radicular and spinal cord arteries along every radicular branch.

The functional arterial anatomy of the cauda equina has been studied by Parke et al., also using an injection technique. ${ }^{1)}$ In their study, by terminating the injection pressure at varying levels, the direction of blood flow in the cauda equina nerve roots was deduced. In the anterior roots of the lumbosacral region of the cord, the one or two anterior medullary feeder arteries (radiculomedullary) carried the afferent flow to the anterior spinal artery, in which it was distributed to the substance of the cord above and below the point of juncture through its numerous sulcal branches and connections with the 


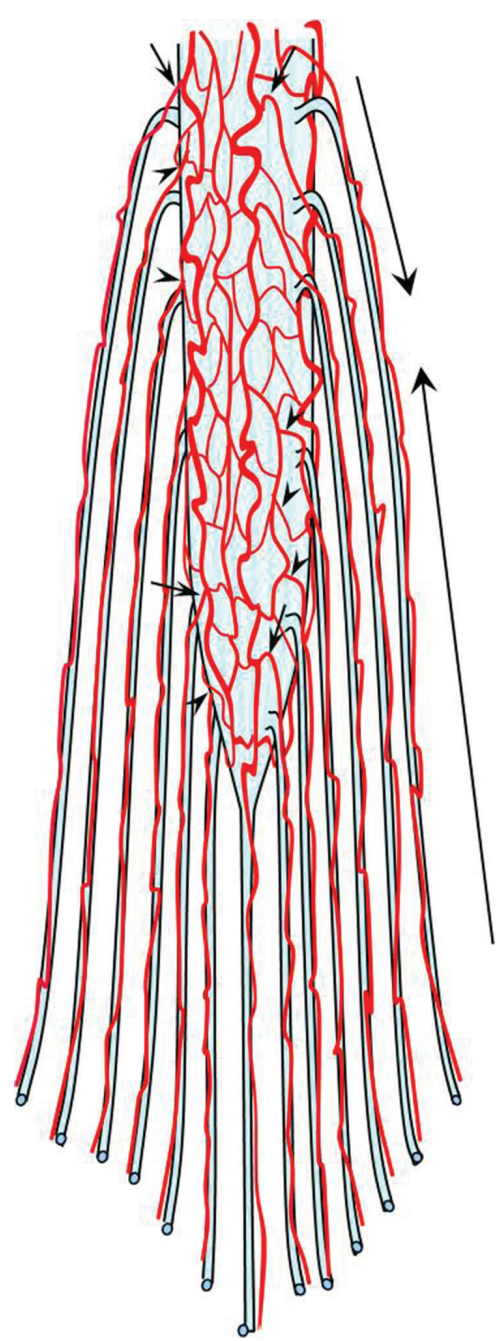

Fig. 3 A schema showing the arterial anatomy of the cauda equina and conus medullaris. On the right-hand side, the arteries of the anterior roots are shown, whereas on the left-hand side, the arteries of the posterior roots are shown. Note the multiple anastomoses among the radicular artery and the spinal cord arteries (arrows) or the vasa corona (arrowheads). The long arrows on the right signify that the proximal one-third of the root is supplied from the radicular artery arising from the arterial plexus of the spinal cord and the distal twothirds from the segmental radicular artery.

vasa corona. From the plexus of the vasa corona, the emerging anterior root fibers received true radicular arteries that supplied proximal one-third of each root (Fig. 3 right, long arrows). The distal two-thirds were then supplied by radicular arteries ascending from the respective segmental sources to anastomose with their superior complement (Fig. 3 right, longer arrow).

In the posterior roots, the distinction between the medullary (radiculopial) and radicular arteries was less marked. Where the posterior spinal arteries coursed just ventral to the emerging rootlets, they gave off the true proximal radicular arteries to the upper one-third of the roots in most cases. However, radiculopial arteries were more numerous in the posterior roots but seldom were of the caliber of the anterior vessels, and the determination of the directions of blood flow was doubtful in some indefinite cases in which the luminal bore approached the size of the true radicular artery.

Taking all the above discussions into account, the arterial supply of the cauda equina as well as in the other spinal cords can be summated as below and as in Fig. 4.

1. The medullary arteries (radiculomedullary and radiculopial) course along their respective roots (Fig. 4, arrows).

2. The distal radicular arteries supply the distal part of the roots and are branches of the segmental artery (Fig. 4, small arrows).

3. The proximal radicular arteries branch from the vasa corona, anterior spinal artery, or posterior spinal artery (Fig. 4, arrowheads).

\section{Venous Anatomy of the Cauda Equina}

Description of the venous anatomy dedicated to the cauda equina is few. The most comprehensive study was also published by Crock et al., and I must refer to their works again. ${ }^{2)}$ Using a retrograde barium sulfate suspension injection via the caval system or the internal vertebral venous plexus, the authors failed to fill the veins of the cauda equina. From these findings as well as in vivo observations by other investigators, they deduced that the venous drainage from the spinal cord was centrifugal. ${ }^{18)}$ Namely, large veins from the spinal cord surface drains via the radicular veins into the extradural system of the internal vertebral venous plexus. The nerve roots of the cauda equina are accompanied by segmentally arranged veins in varying size that are derived from either the anterior or posterior longitudinal venous trunk.

\section{Vascular Anatomy of the Filum Terminale (Fig. 5)}

The vascular anatomy of the intradural filum terminale has been studied by Djindjian et al. in their landmark work in $1988{ }^{3}{ }^{3}$ The filum terminale was constantly supplied by a single artery, the artery of the film terminale, which arose from the termination of the anterior spinal artery (Fig. 5, small arrows). Since there are no nerves arising distal to the conus, and the filum terminale being the last 


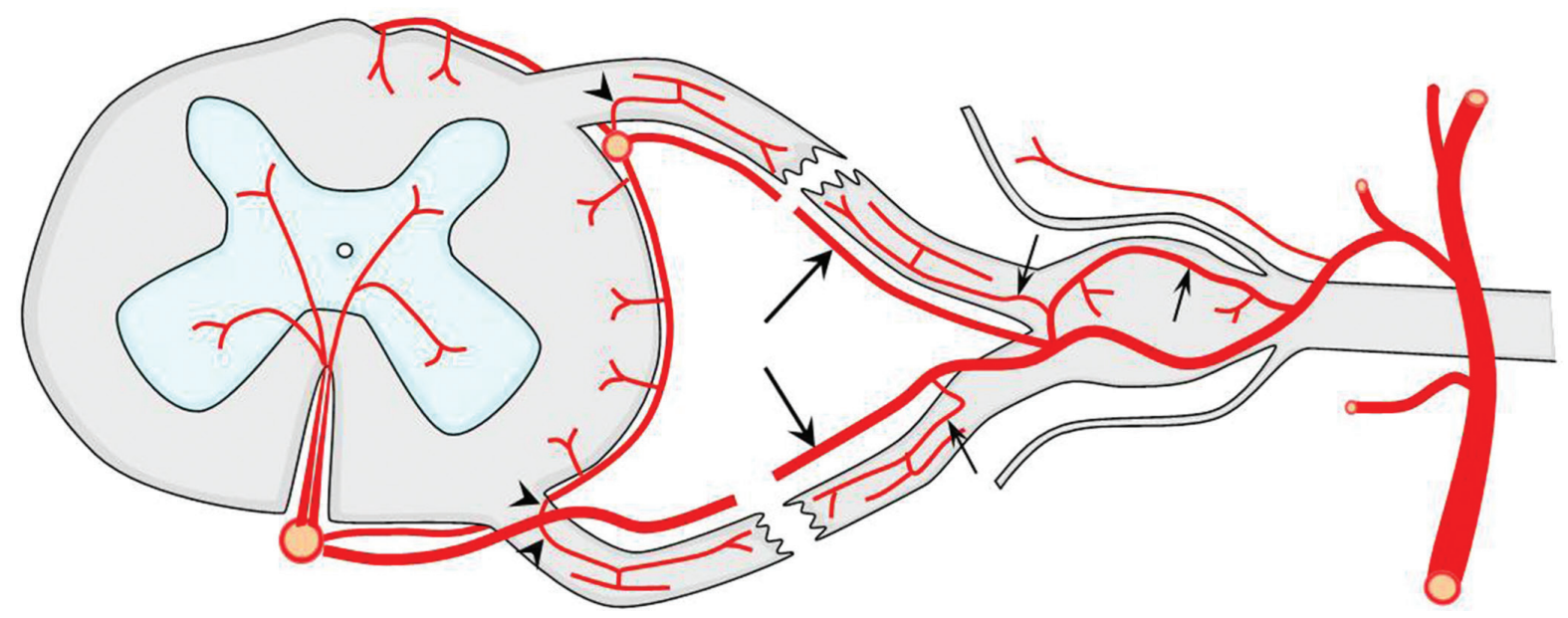

Fig. 4 A schema illustrating all the possible arterial structures of the spinal nerve roots that may be applicable to all segmental levels. The radiculomedullary and radiculopial arteries (arrows) course along their respective nerve roots to supply the spinal cord. The segmental arteries give rise to the distal radicular arteries to supply the distal part of the roots (small arrows). The anterior and posterior spinal arteries or the vasa corona give rise to the radicular arteries in the proximal nerve roots (arrowheads).

spinal cord structure, there cannot be any low lumbar or sacral artery contribution to the filum. ${ }^{19)}$ However, Djindjian et al. made a noteworthy observation on the peripheral vascular supply from the filum terminale artery. ${ }^{3)}$ They demonstrated arterioles arising from the proximal portion of the filum terminale artery supplying the coccygeal nerve roots adherent to the filum terminale (Fig. 5, arrowheads). In certain pathological condition, this may serve as a potential collateral pathway.

A vein of the filum terminale was also constantly found on the ventral aspect of the filum, dorsal to the filum terminale artery (Fig. 5, arrows). While maintaining a constant caliber, the vein was continuous with the anterior spinal vein cranially, and caudally traversed the dural sac toward the sacral venous plexus and the internal iliac vein. From their observations, they inferred that the vein functioned as a true venous axis, capable of draining bidirectionally: cranial and caudal.

The vascular pattern of the filum terminale may be reasonable in light of the view that the filum terminale is embryologically the dediferrentiated spinal cord.

\section{Implications of the Vascular Anatomy on the Vascular Lesions in the Cauda Equina}

The cauda equina is composed of the lumbosacral and coccygeal nerve roots and the filum terminale. Naturally, intradural vascular lesion in this region forms on either structure. As discussed so far,

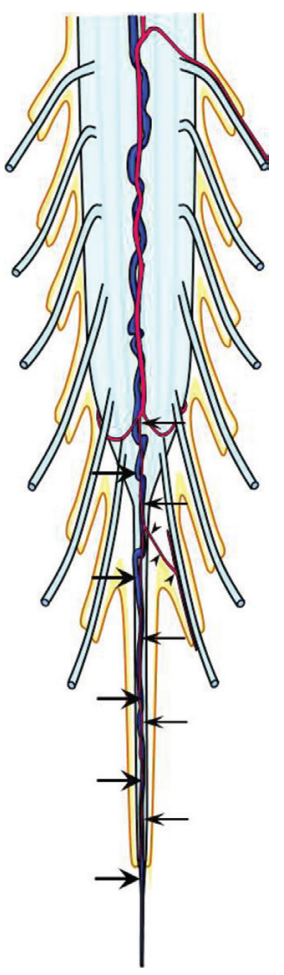

Fig. 5 A schema of the vascular anatomy of the filum terminale. The artery of the filum terminale originates from the termination of the anterior spinal artery (arrows). The filum artery may give rise to arterioles which supply the coccygeal nerve roots adherent to the filum terminale (arrowheads). The vein of the filum terminale exists constantly on the ventral aspect of the filum, behind the artery (large arrows). The vein is continuous from the anterior spinal vein to the sacral dural sac, where it traverses the dura to join the sacral venous plexus. 
the arterial supply to filum terminale is derived from the artery of the filum, which is the caudal extension of the anterior spinal artery. Therefore, vascular lesion of the filum most likely receives its arterial supply from the anterior spinal artery. Accordingly, one must strive to visualize the anterior spinal artery in diagnosing a vascular lesion of the filum terminale. In very rare occasions, both the anterior spinal artery and the branch of the lateral sacral artery may supply a vascular lesion of the filum terminale. ${ }^{20)}$ This vascular pattern is contradicted in the textbook of Lasjaunias et al. for the reason that there cannot be any lumbar or sacral artery contribution to the filum. ${ }^{19)}$ They state that any cauda equina lesion fed by an arterial branch of the basket and an ascending contribution from the lumbar or sacral arteries has to be radicular. Theoretically, this is true. However, as Djindjian et al. have demonstrated, an arterial pathway from the coccygeal nerve artery to the filum terminale could exist. ${ }^{3)}$ The author of this review presumes that this pathway must have played a role in the case of Jin et al. ${ }^{20)}$

On the other hand, vascular lesion of the nerve root of the cauda equina will be expected to be supplied by the radicular artery arising from the lumbar, middle and lateral sacral, or lumbosacral arteries. Because of the functional anastomoses between the radicular artery and the spinal cord artery at the conus medullaris, and the evidence that the proximal one-third of the nerve root is supplied by a radicular artery arising from the plexus of the conus, an anterior or posterior spinal artery can be a feeder to the lesion. Thus, vascular lesion in the cauda equina radicular nerve may be supplied by a radicular artery or a spinal cord artery, or both arteries.

Table 1 summarizes the potential arterial supply to the cauda equina vascular lesion.

\section{Conclusion}

The vascular anatomy of the cauda equina was reviewed in conjunction with its implication on the vascular lesions in this region. The lumbosacral and coccygeal nerve roots and the film terminale constitute the intradural cauda equina structure. Each nerve root is supplied by the radicular artery arising from the corresponding lumbar, medial and lateral sacral, or iliolumbar artery. The proximal radicular artery anastomoses with the spinal cord artery in the conus medullaris and the nerve roots in the proximal portion may be supplied craniocaudally. The filum terminale is supplied by the artery of the filum that is a direct extension of the anterior spinal
Table 1 Possible arterial supply to the vascular lesions in the cauda equina

\begin{tabular}{lll}
\hline Artery & Spinal cord & Radicular \\
\hline Filum terminale lesion & $\bigcirc$ ASA & $\triangle$ coccygeal \\
Radicular lesion & $\bigcirc$ ASA, PSA, & $\bigcirc$ \\
& vasa corona & \\
\hline
\end{tabular}

(O): most likely feeder, $\bigcirc$ : likely feeder, $\triangle$ : rare feeder, ASA: anterior spinal artery, PSA: posterior spinal artery.

artery, which may be related to its developmental origin. Vascular lesions on the filum terminale or the nerve roots of the cauda equina may have a variety of arterial supplies derived from either the spinal cord or radicular artery, or both. Therefore, both the spinal cord artery, most likely the artery of Adamkiewicz, and the radicular arteries must be examined in the angiographic study of a vascular lesion in the cauda equina.

\section{Acknowledgments}

The author thanks Ms. Hisako Namba for preparing all the illustrations in this review.

\section{Conflicts of Interest Disclosure}

None.

\section{References}

1) Parke WW, Gammell K, Rothman RH: Arterial vascularization of the cauda equina. J Bone Joint Surg Am 63: 53-62, 1981

2) Crock HV, Yamagishi M, Crock MC: The Conus Medullaris and Cauda Equina in Man. An Atlas of the Arteries and Veins. Wien-New York, Springer-Verlag, 1986

3) Djindjian M, Ribeiro A, Ortega E, Gaston A, Poirier J: The normal vascularization of the intradural filum terminale in man. Surg Radiol Anat 10: 201-209, 1988

4) Sadler TW: Langman's Medical Embryology, ed 11. Baltimore, Lippincott Williams \& Wilkins, 2010, pp 67-70

5) Saitsu H, Shiota K: Involvement of the axially condensed tail bud mesenchyme in normal and abnormal human posterior neural tube development. Congenit Anom (Kyoto) 48: 1-6, 2008

6) O'Rahilly R, Müller F: Neurulation in the normal human embryo. Ciba Found Symp 181: 70-82; discussion 82-89, 1994

7) Le Douarin NM, Teillet MA, Catala M: Neurulation in amniote vertebrates: a novel view deduced from the use of quail-chick chimeras. Int J Dev Biol 42: 909-916, 1998 
8) O’Rahilly R, Müller F: Somites, spinal Ganglia, and centra. Enumeration and interrelationships in staged human embryos, and implications for neural tube defects. Cells Tissues Organs (Print) 173: 75-92, 2003

9) Kunitomo K: The development and reduction of the tail and of the caudal end of the spinal cord in the human embryo. Contribut Embryol 8: 161-198, 1918

10) Streeter GL: Factors involved in the formation of the filum terminale. Am J Anat 25: 1-12, 1919

11) Choi BH, Kim RC, Suzuki M, Choe W: The ventriculus terminalis and filum terminale of the human spinal cord. Hum Pathol 23: 916-920, 1992

12) Harmeier J: The normal histology of the intradural filum terminale. Arch Neurol Psychiatry 29: 308-316, 1933

13) Tarlov IM: Structure of the filum terminale. Arch Neurol Psychiatry 40: 1-17, 1938

14) Adamkiewicz A.: Die Blutgefäße des menschlichen Rückenmarks. II. Die Gefäße der Rückenmarkesoberfläche. Sitz. ber. Akad. Wiss. Wien, Math.-nat. Kl. 85: 101-130, 1882

15) Kadyi H: Über die blutgefäße des menschlichen rückenmarks. Lemberg, Gubrynowicu \& Schmidt, 1889
16) Tanon L: Les artères de la moelle dorso-lombaire. Paris, Vigot Frères, 1908

17) Lazorthes G, Gouaze A, Zadeh JO, Santini JJ, Lazorthes Y, Burdin P: Arterial vascularization of the spinal cord. Recent studies of the anastomotic substitution pathways. J Neurosurg 35: 253-262, 1971

18) Doppmann JL, Di Chiro G, Ommaya AK: Selective Arteriography of the Spinal Cord. St. Louis, W.H. Green, 1969

19) Lasjaunias P, Berenstein A, ter Brugge KG: Surgical Neuroangiography, Volume 1, Clinical Vascular Anatomy and Variations, ed 2. Berlin Heidelberg New York, Springer-Verlag, 2001, pp 132-134

20) Jin YJ, Kim KJ, Kwon OK, Chung SK: Perimedullary arteriovenous fistula of the filum terminale: case report. Neurosurgery 66: E219-E220; discussion E220, 2010

Address reprint requests to: Katsunari Namba, $\mathrm{MD}, \mathrm{PhD}$, Center for Endovascular Therapy, Division of Neuroendovascular Surgery, Jichi Medical University, 3311-1 Yakushiji, Shimotsuke, Tochigi 329-0498, Japan. e-mail: knamba@jichi.ac.jp 